\title{
Adverse drug reactions
}

In this clinical update (BMJ 2018;363:k4051, doi:10.1136/bmj. k4051) by Robin Ferner and Patricia McGettigan, figure 3

(Algorithm for patients on when and how to report adverse drug reactions) wrongly advises patient to "Report your suspicion to the National Medicines Regulator ... or European Medicines Agency." In fact, patients may report suspected adverse drug reactions directly to their national medicines regulators but not directly to the European Medicines Agency.

In addition, the authors' list of competing interests should have included the statement: "PM is on part-secondment at the European Medicines Agency." 